\title{
Intravitreal Injection Bevacizumab: Main Indications
}

\section{Babita Gurung, Eliya Shrestha, Hara Maya Gurung, Hari Bikram Adhikari, Anju Gurung, Krishna Gurung and Pawan Baral*}

Himalaya Eye Hospital, Pokhara, Nepal

*Corresponding Author: Pawan Baral, Himalaya Eye Hospital, Pokhara, Nepal.
Received: June 17, 2021

Published: July 30, 2021

(C) All rights are reserved by Babita Gurung., et al.

\begin{abstract}
Purpose: To study the main indications of intravitreal injection Bevacizumab in Himalaya Eye Hospital, Pokhara, Nepal.

Methods: A retrospective study of the patients who received intravitreal injection Bevacizumab (IVB) from July 2019 to December 2020 was carried out at Himalaya Eye Hospital, Pokhara, Nepal. Patients who received only IVB were included in the study. Patients who received IVB along with other intraocular surgery were excluded from the study.

Results: 468 patients received IVB during this study period. 281 were males and 187 were females. Diabetic retinopathy was found to be the most common indication for IVB followed by branch retinal vein occlusion, age related macular degeneration, central retinal vein occlusion and others. There was no recorded complications of IVB during this period.

Conclusion: Diabetic retinopathy is the most common indication for IVB followed by branch retinal vein occlusion. The technique of intravitreal injection is considered safe.
\end{abstract}

Keywords: Bevacizumab; Indication; Diabetic Retinopathy; Safe; Nepal

\section{Abbreviations}

BRVO: Branch Retinal Vein Occlusion; CNV: Choroidal Neovascularization; CRVO: Central Retinal Vein Occlusion; DME: Diabetic Macular Oedema; HEH: Himalaya Eye Hospital; IVB: Intravitreal Injection Bevacizumab; PDR: Proliferative Diabetic Retinopathy; RVO: Retinal Vein Occlusion; VEGF: Vascular Endothelial Growth Factor

\section{Introduction}

Intravitreal injection of drugs have become a part of routine ophthalmic procedure. Besides the various intravitreal injection of antimicrobials and steroids, Anti-Vascular Endothelial Growth Factor (Anti-VEGF) drugs like Bevacizumab (Avastin) has gained increasing acceptance in ocular therapeutic management. Since May 2005, off-label use of intravitreal injection Bevacizumab (Avastin;
Genentech Inc., South San Francisco, CA, USA) have been used for the treatment of neovascular and exudative ocular diseases [1]. Since then many anti-VEGF injections have been developed. Among those injections, Bevacizumab is widely accepted in our country. Bevacizumab is being used in various conditions like choroidal neovascularization ( $\mathrm{CNV}$ ) secondary to age related macular degeneration (AMD), pathological myopia, macular edema secondary to branch or central retinal vein occlusion (BRVO or CRVO), proliferative diabetic retinopathy (PDR), retinopathy of prematurity (ROP) [2]. Despite its wide usage, the serious adverse effects of injection Bevacizumab is low [3].

Himalaya Eye Hospital (HEH), in Pokhara Nepal is one of the several tertiary eye hospitals in Nepal. HEH is situated in Kaski district and serves over 2 million (9.06\% of the country's population) 
population of Gandaki Province. At HEH IVB has been used for over five years for the treatment of ocular conditions like BRVO, CRVO, Macular oedema, CNV, PDR etc [4].

\section{Aim of the Study}

The aim of this study is to enumerate the main indications of this widely used IVB at HEH. It is likely that the information of the main indications for IVB at HEH will provide basic information about the patterns of ocular diseases indicative of IVB in Gandaki Province.

\section{Materials and Methods}

This is a retrospective study conducted at HEH, Pokhara, Nepal. Eighteen months data of the patients from July 2019 to December 2020 were collected from the database of the operation theatre of the hospital.

Inclusion criteria: Patients who received only IVB.

Exclusion criteria: Patients who received IVB together with other intraocular surgery.

A total of 468 patients received IVB. The procedure of IVB practiced in the hospital is as follows: After complete ocular examinations, patients who may benefit from IVB are selected and counselled for IVB. In the counselling process the informed consent is obtained from the patients undergoing IVB procedure. All injections are performed in the operation theatre under strict aseptic precautions. Under topical anaesthesia injection Bevacizumab (Avastin) $1.25 \mathrm{mg} / 0.05 \mathrm{ml}$ is injected $3.5 \mathrm{~mm}$ from the limbus superotemporally. The drug used for topical anaesthesia is proparacaine $0.5 \%$ instilled 1 drop at a time in the interval of 15 minutes. The needle is directed towards the center of the vitreous cavity. The patients are given one tablet of oral acetazolamide $250 \mathrm{mg}$ tablet stat soon after the procedure. Moxifloxacin eye drop $0.3 \%$ is prescribed to be instilled 1 drop at a time for 4 times a day for one week and tablet Ciprofloxacin 500mg to be taken twice a day for 3 days. The patients are asked to report immediately if they experience any of the symptoms like blurring of vision, pain, redness and discomfort. Otherwise in normal conditions the patients are followed up after one month of injection.

\section{Data collection and statistical analysis}

The data was initially collected in the standard form developed for the study. The form included variables for patients' demograph- ic details including age, gender, ethnicity, address, eye, and the condition for which the IVB was planned. The data collected in the forms was then entered in the Microsoft Excel 2013 and analyzed in same.

\section{Results}

This retrospective study showed that a total of 468 patients received IVB during the study period. Of the total, 281 (60.04\%) were males and 187 (39.96\%) were females. The mean age of the patients was $62.98( \pm 13.34)$ years of age and the patients' age ranged from 28 to 95 years old. Mean age being 63 years old.

Diabetic retinopathy (DR) was found to be the main indication for IVB in the hospital followed by Branch retinal vein occlusion (BRVO) and is summarized in table 1.

\begin{tabular}{|l|c|}
\hline \multicolumn{1}{|c|}{ Condition } & \multicolumn{1}{c|}{$\begin{array}{c}\text { Number of Cases/ } \\
\text { Avastin injections }\end{array}$} \\
\hline Diabetic Retinopathy & 133 \\
\hline Branch Retinal Vein Occlusion & 102 \\
\hline Age Related Macular Degeneration & 79 \\
\hline Central Retinal Vein Occlusion & 47 \\
\hline Choroidal Neovascularization & 44 \\
\hline Vitreous Haemorrhage & 26 \\
\hline Central Serous Retinopathy & 21 \\
\hline Maculopathy & 11 \\
\hline $\begin{array}{l}\text { Polypoidal Choroidal Vasculopa- } \\
\text { thy }\end{array}$ & 3 \\
\hline Retinal Hemorrhage & 2 \\
\hline Total & 468 \\
\hline
\end{tabular}

Table 1: Indications for intravitreal bevacizumab injections.

Left eye was found more affected than right eye. 240 patients received injection in their left eye while 228 patients received in their right eye.

Out of the total 11 districts in the Gandaki Province, the maximum number of IVB were performed in the patients from Kaski district followed by Syangja and Tanahun. The details of IVB performed per districts is provided in table 2 .

\section{Discussion}

Diabetic retinopathy topped the list of indications for IVB in our hospital. This is evident from the fact that almost all developing 


\begin{tabular}{|l|c|}
\hline \multicolumn{1}{|c|}{ District } & Number of Avastin injections \\
\hline Kaski & 308 \\
\hline Syangja & 57 \\
\hline Tanahun & 46 \\
\hline Myagdi & 20 \\
\hline Parbat & 14 \\
\hline Baglung & 7 \\
\hline Gorkha & 6 \\
\hline Lamjung & 6 \\
\hline Others & 4 \\
\hline Total & 468 \\
\hline
\end{tabular}

Table 2: Number of Intravitreal bevacizumab injections performed per district.

countries in the Western Pacific region and South Asia have shown significant rise in the number of diabetic population [5] and Diabetic Macular Edema (DME) being the cause for visual impairment [6]. Mahat P., et al. have shown the DME as the major cause for visual impairment which improved with IVB [7]. BRVO was found the second most common condition after diabetic retinopathy in this study which matches the conclusion drawn by Thapa R., et al. in her research [8]. Neovascular condition, secondary to Age related macular degeneration ranked third among the indications for IVB. Off-label use of IVB was used for this type of neovascular and other exudative conditions since May 2005 [1]. Despite its Off-label status, IVB has gained increasing popularity for treatment of ocular conditions and considered safe as quoted in many studies including the one done at Tilganga Institute of Ophthalmology by Pradhan E., et al [9]. No recorded complications of IVB was found in our study.

\section{Conclusion}

Diabetes Mellitus, an emerging medical condition, has both social and economic impacts in both developed and developing countries. Diabetic retinopathy is one of the systemic adverse effects of this condition. So, this study too found diabetic retinopathy as the most common indication for IVB followed by retinal vein occlusion disease and age related macular degeneration. Good glycaemic control and routine ocular examination can prevent from this blinding condition - diabetic retinopathy.

\section{Acknowledgements}

The authors would like to acknowledge the support provided by Himalaya Eye Hospital in conducting this study.

\section{Conflict of Interest}

None.

\section{Bibliography}

1. Artunay 0., et al. "Incidence and management of acute endophthalmitis after intravitreal Bevacizumab (Avastin) injection". Eye 23 (2009): 2187-2193.

2. Afarid M., et al. "Intravitreal injection of Bevacizumab: Review of our previous experience". Iranian Journal of Pharmaceutical Research 17 (2018): 1093-1098.

3. Jager R D., et al. "Risk of intravitreous injection: A comprehensive review". Retina 24 (2004): 676-698.

4. Ministry of Federal Administration and General Administration (MoFAGA). Brief Introduction of Gandaki Province (2021).

5. Nanditha A., et al. "Diabetes in Asia and the Pacific. Implications for the global epidemic". Diabetes Care 39 (2016): 472-485.

6. Jan S., et al. "Intravitreal Bevacizumab: Indications and complications". Journal of Ayub Medical College, Abbottabad 28.2 (2016): 364-368.

7. Mahat P., et al. "Evaluation of intravitreal Bevacizumab (Avastin) in patients with diabetic macular edema". Nepalese Journal of Ophthalmology 12.24 (2020): 236-244.

8. Thapa R., et al. "Demographic characteristics, patterns and risk factors for retinal vein occlusion in Nepal: a hospital-based case-control study". Clinical and Experimental Ophthalmology 38 (2010): 583-590.

9. Pradhan E Duwal S., et al. "Acute endophthalmitis after intravitreal Bevacizumab injection at the tertiary centre in Nepal". Nepalese Journal of Ophthalmology 10.19 (2018): 107-110.

\section{Volume 4 Issue 8 August 2021}

(C) All rights are reserved by Babita Gurung., et al. 